\title{
Características de las tutorías del Centro de Lectura y Escritura de la Universidad Autónoma de Occidente: ¿Qué muestran los registros de atención?
}

Claudia Alexandra Roldán Morales'

Karina Alejandra Arenas Hernández ${ }^{2}$

Universidad Autónoma de Occidente- Cali, Colombia

Artículo de Investigación

Recibido: Marzo 30 de 2016 Aprobado: Mayo II de 2016

\section{Resumen}

En el presente artículo se socializan los principales hallazgos de una investigación que se adelantó en el primer semestre del 20 I 5 para analizar las características de las tutorías llevadas a cabo en el Centro de Lectura y Escritura de la Universidad Autónoma de Occidente, con base en la revisión de los registros escritos de atención de los tutores. Entre los principales resultados, se evidencia que existen diferentes percepciones de la tutoría, que van desde las visiones remediales hasta las que se ocupan de fomentar el desarrollo de estrategias por parte de los asesorados.

Palabras clave: centro de escritura, clases de tutoría, estrategias de asesoría, tutor de escritura, registros de atención, tutorados.

I Licenciada en Literatura, Magíster en Lingüística y Español, Doctora en Educación. Jefe del Departamento de Lenguaje de la Universidad Autónoma de Occidente. Correo electrónico: caaroldan@uao.edu.co

2 Fonoaudióloga, Magíster en Lingüística y Español. Docente Departamento de Lenguaje de la Universidad Autónoma de Occidente. Correo electrónico: kaarenas@uao.edu.co 


\title{
Characteristics of tutorial sessions at Centro de Lectura y Escritura de la Universidad Autonoma de Occidente: What do the attention records show?
}

\begin{abstract}
The current paper aims at socializing the main findings of a piece of research carried out in the first semester of 2015 with the purpose of analyzing the characteristics of the tutorial sessions held at Centro de Lectura y Escritura de la Universidad Autonoma de Occidente based on the perusal of the written records regarding tutors' attention. Among the main findings, it is evidenced that there are different perceptions of tutorial sessions, which go from remedial instances to those of enhancing the development of strategies in the tutees.
\end{abstract}

Key words: writing center, tutorial sessions, tutorial strategies, writing tutor, attention records, tutees

\section{Características das tutorias dos centros de Leitura e Escrita na Universidade Autônoma de Ocidente: o que mostram os registros de atenção?}

\begin{abstract}
Resumo
No presente artigo são socializados os principais achados de uma pesquisa que foi realizada no primeiro semestre de 2015 para analisar as características das tutorias levadas a cabo no Centro de Leitura e Escrita da Universidade Autônoma de Ocidente, com base na revisão dos registros escritos de atenção dos tutores. Entre os principais resultados se evidencia que existem diferentes percepções da tutoria, que vão das visões remediais até as que se ocupam de fomentar o desenvolvimento de estratégias por parte dos assessorados.
\end{abstract}

Palavras chave: centros de escrita, aulas de tutoria, estratégias de assessoria, tutor de escrita, registros de atenção, tutorados.

\section{Introducción}

El Centro de Lectura y Escritura de la Universidad Autónoma de Occidente - CELEE- fue creado por el Departamento de Lenguaje de la Facultad de Comunicación Social y se concibe como una estrategia con la que se pretende contribuir a la excelencia académica de la institución, mediante el funcionamiento de un espacio que propicia el acompañamiento, tanto a estudiantes como a docentes, en el proceso de comprensión y producción de textos escritos académicos y profesionales. Una de las formas de funcionamiento de dicho espacio es a través de asesorías personalizadas, en las que se brindan los apoyos necesarios para que quienes asistan conozcan y apliquen estrategias que les permitan desempeñarse de manera adecuada en los procesos de lectura, escritura y oralidad en el contexto universitario. En promedio, el Centro funciona 36 horas por semana durante 13 semanas en un semestre. 
El equipo de tutores está conformado por 5 docentes (2 hora cátedra y 3 de tiempo completo) y 3 estudiantes ( I del programa PILO3 y 2 monitoras académicas). De cada sesión de asesoría se debe dejar un registro escrito en rejillas que se encuentran en un espacio virtual de Google Drive al que todos los tutores pueden acceder en cualquier momento. La información que se pide diligenciar está relacionada con los nombres completos de los estudiantes que consultan, las asignaturas y los programas académicos a los que pertenecen, los motivos generales de consulta y un seguimiento del tutor; en este último espacio se debe hacer una descripción detallada del desarrollo de la sesión de tutoría.

Hasta el momento, estos registros se habían usado para identificar los promedios de atención en el espacio y los motivos generales de consulta; sin embargo, no se había hecho un análisis riguroso que permitiera evidenciar las características del acompañamiento que hacen los tutores. En el primer semestre del año 20 I5, se consolidó el equipo coordinador del Centro, que decidió iniciar el proceso de análisis de dichos registros, debido a que representan un importante insumo para identificar un panorama general de las necesidades en términos de reorientación de algunas dinámicas académicas e investigativas del Centro.

\section{Marco conceptual}

Acto seguido, se expone la revisión general de algunos referentes teóricos que ilustraron el análisis de los datos tomados para esta experiencia.

\section{Clases de tutoría ${ }^{4}$}

Para comprender las generalidades de lo que se presenta en este trabajo, es fundamental mencionar cuáles son los elementos que definen las tutorías, como los espacios en los que se concretan diferentes apoyos para la comprensión y producción de textos.

Brown ${ }^{5}$, apoyada en Donal McAndrew and Thomas Reigstad, menciona tres clases de tutoría: I) Centrada en el estudiante, 2) Centrada en el tutory 3) Colaborativa. Las diferencias entre estas están dadas por los roles que asume cada uno de los participantes. En la tutoría centrada en el estudiante, él toma la iniciativa, formula las inquietudes que tiene sobre la escritura del texto y acoge las recomendaciones o sugerencias que hace el tutor, quien se encarga de responder a las diversas preguntas. En la segunda clase de tutoría, que es centrada en el tutor, este domina la situación ya que es quien habla, hace las preguntas y el papel del estudiante es el de responder; el tutor lee el texto y señala

3 El programa PILO es un servicio de crédito financiero a largo plazo que permite a un estudiante ingresar a primer semestre o sostenerlo en su programa académico, privilegiando su excelencia académica.

4 El marco teórico ha sido producto de un equipo de trabajo colegiado del Departamento de Lenguaje conformado por los docentes María Mercedes Chacón, Adriana de La Rosa, Karina Arenas, Viviana Arango y Claudia Roldán.

5 Brown, K. Breaking into the tutor"s toolbox: an investigation into strategies used writing center tutorials. Dissertation. University of Louisville, 2008. 
los errores. Se critica esta forma de proceder ya que vulnera una de las premisas de la enseñanza no directiva que es respetar la propiedad e individualidad del autor del texto.

Por su parte, en una tutoría colaborativa, "tutor y estudiante comparten la resolución de los problemas y la toma de decisiones"6. Para lograr una ubicación desde este enfoque es fundamental que los tutores tengan claro que el texto objeto de la tutoría es responsabilidad del estudiante y no del tutor. Es valiosa la recomendación de Brown, en la que señala que la experiencia de la mayoría de los estudiantes universitarios es con métodos directivos y autoritarios y no se sienten cómodos con modelos pedagógicos colaborativos que los ubiquen en el lugar de responsables y dueños de su texto, ya que esperan que el tutor les indique qué y cómo deben reescribirlo. De la discusión anterior se desprende el método no directivo como uno de los modelos pedagógicos en los que se inscribe la enseñanza de la escritura. Desde este es importante cuestionar la concepción de "texto ideal" del tutor que asesora en el centro de escritura, para generar una reflexión que lleve a relativizar esta idea porque lo que él espera del texto puede entrar en conflicto con los objetivos y las expectativas que tienen los estudiantes sobre su trabajo de escritura. En este sentido, se sugiere que en la tutoría se debe recoger de manera activa la percepción del estudiante frente a su texto, su proceso y promover su participación constante.

Al respecto y como complemento de lo anterior, Alzate-Medina y Peña-Borrero proponen una distinción entre las características tradicionales de las tutorías de escritura y la evolución que se ha presentado del concepto de estas. La diferencia básica es que la concepción tradicional buscaba solucionar problemas en los textos, mientras que la más reciente promueve un espacio de apoyo al estudiante en el que pueda desarrollar estrategias propias de solución de problemáticas, un ejercicio metacognitivo antes que el de revisión textual.

Desde estas aproximaciones, es claro que los nuevos enfoques de las tutorías implican un cambio en el rol del tutor, que debe orientar la identificación de herramientas por parte del tutorado para que se apropie de su trabajo y pueda generar preguntas y respuestas. De acuerdo con los autores mencionados, "el tutor pasa a ser un facilitador que conoce el potencial del estudiante, sabe escuchar, guía, orienta, presenta alternativas, le ofrece herramientas para que desarrolle exitosamente su trabajo y sabe reconocer oportunamente sus logros"1. Así pues, de acuerdo con Maceira9 y Clark ${ }^{10}$, se podría afirmar que en un centro de escritura los apoyos deben trascender las acciones remediales para que las tutorías se conviertan y posicionen como una estrategia que hace aportes al desarrollo de la creatividad, la capacidad de investigación, el aprendizaje autónomo y las competencias comunicativas de los estudiantes asesorados.

6 Íbid, pág. 19.

7 Alzate, G., y Peña, L. Universitas Phychologica. La tutoría entre iguales: una modalidad para el desarrollo de la escritura en la educación superior, 9, 2010 págs. 123- 138.

8 Íbid, pág. 125.

9 Citado en Alzate, G., y Peña (20 l0).

10 Clark, I. Dialogue in the Lab-Conference: Script Writing and the Training of Writing Lab Tutors, 1982. Recuperado el 10 de mayo de 2015 de http://casebuilder.rhet.ualr.edu/wcrp/publications/wcj/wcj2.I/wcj2. I_clark.pdf 


\section{Metodología}

Esta investigación es de corte cualitativo. El valor de estos datos reside en que revela hechos, acontecimientos e ideas que emanan de la realidad y que, al ser parte de un contexto particular, son singulares. Por esto, no existe un modelo estandarizado para el análisis de los datos; sin embargo, hay unas tareas y recomendaciones para su manejo, organización y presentación, así como para la extracción final de las conclusiones".

El propósito de esta investigación fue caracterizar el tipo de tutoría y las estrategias que usan los tutores y sus formas de brindar los apoyos. La experiencia se documenta a través de los registros escritos realizados por los tutores del Centro de Lectura y Escritura, correspondientes a los últimos tres meses del periodo académico del 2014 y los tres últimos del 2015. Estos periodos se seleccionaron teniendo en cuenta que fueron los de mayor afluencia de estudiantes al espacio. En total se revisaron los registros escritos de cinco profesores y tres estudiantes tutores. Los primeros son profesores que orientan clase en el Departamento de Lenguaje, en los cursos de 'Comunicación Oral y Escrita' y 'Expresión Oral y Escrita' en el primer semestre de formación, y, los segundos, tres estudiantes que cursan entre séptimo y noveno semestre de programas de la Facultad de Comunicación Social y tienen altos niveles de desempeño en lectura y escritura.

El modelo de análisis de este tipo de datos implica tres tareas. La primera es la reducción de datos, la segunda es la organización y presentación, y la tercera es la interpretación y verificación. En este orden de ideas, se procedió a ordenar la información y a establecer categorías y códigos para el análisis. Por lo anterior, se determinaron categorías temáticas para analizar cuestiones primordiales que han surgido del proceso de investigación; para tal fin, se eligieron ciertos fragmentos que estuvieran relacionados con las categorías.

A continuación, se expone una tabla con las macrocategorías, categorías y códigos, y una descripción del significado que tienen para el caso de esta investigación.

\begin{tabular}{|l|l|l|l|l|l|}
\hline & \multicolumn{1}{|c|}{ Macrocategoría } & & \multicolumn{1}{c|}{ Categorías } & Código & \multicolumn{1}{c|}{ Definición } \\
\hline A & Aspectos reiterados & I & Generalidades & G & $\begin{array}{l}\text { Se refiere a aquellos aspectos que se presentan de } \\
\text { manera reiterada en los registros de los tutores. }\end{array}$ \\
\hline B & $\begin{array}{l}\text { Tendencia de tipo de } \\
\text { asesoría }\end{array}$ & 2 & Centrada en el tutor & CT & $\begin{array}{l}\text { El tutor domina la situación, es quien habla, hace las } \\
\text { preguntas, lee el texto y señala los errores. }\end{array}$ \\
\cline { 3 - 6 } & 3 & Colaborativa & C & $\begin{array}{l}\text { El tutor recoge la percepción del estudiante frente } \\
\text { su texto, su proceso y promueve su participación } \\
\text { constante. }\end{array}$ \\
\hline
\end{tabular}

\footnotetext{
I I Rodríguez, G., Gil Flores, J. y García Jiménez, E. Metodología de la investigación Cualitativa. Málaga: Aljibe, 1999.
} 


\begin{tabular}{|c|c|c|c|c|c|}
\hline & Macrocategoría & & Categorías & Código & Definición \\
\hline \multirow{8}{*}{ C } & \multirow{8}{*}{$\begin{array}{l}\text { Estrategias orientación } \\
\text { de las asesorías }\end{array}$} & 4 & Revisión de la consigna & $\mathrm{RC}$ & $\begin{array}{l}\text { Atañe a la identificación del tipo de demandas que } \\
\text { aparecen en las consignas, para orientar las sesiones } \\
\text { de asesoría. }\end{array}$ \\
\hline & & 5 & Revisión de material & RMT & $\begin{array}{l}\text { Se refiere a la recomendación que hacen los tuto- } \\
\text { res sobre material para ampliar algunos aspectos o } \\
\text { aclarar dudas. }\end{array}$ \\
\hline & & 6 & $\begin{array}{l}\text { Conexión entre la tutoría } \\
\text { y la asignatura }\end{array}$ & CTA & $\begin{array}{l}\text { Alude al trabajo colaborativo entre el Centro y el } \\
\text { profesor que propone la actividad al estudiante. }\end{array}$ \\
\hline & & 7 & $\begin{array}{l}\text { Explicación breve de algún } \\
\text { aspecto }\end{array}$ & EA & $\begin{array}{l}\text { Describe explicaciones sobre aspectos puntuales } \\
\text { que debe hacer el tutorado. }\end{array}$ \\
\hline & & 8 & Formulación de preguntas & FP & $\begin{array}{l}\text { Alude a la pregunta como estrategia para guiar la } \\
\text { discusión y potenciar la reflexión de los estudiantes } \\
\text { sobre los procesos de lectura y escritura. }\end{array}$ \\
\hline & & 9 & $\begin{array}{l}\text { Definición de un plan de } \\
\text { escritura }\end{array}$ & DPE & $\begin{array}{l}\text { Está relacionado con la representación de la tarea, el } \\
\text { plan de composición y la generación y organización } \\
\text { de las ideas. }\end{array}$ \\
\hline & & 10 & $\begin{array}{l}\text { Ofreciendo modelos de } \\
\text { textos }\end{array}$ & OMT & $\begin{array}{l}\text { Alude a ofrecer a los estudiantes modelos para que, } \\
\text { a partir de estos, sean capaces de aplicarlos en di- } \\
\text { ferentes situaciones. }\end{array}$ \\
\hline & & II & Lectura en voz alta & LVA & $\begin{array}{l}\text { Refiere a la lectura en voz alta que hace el estudiante, } \\
\text { para favorecer la identificación de ideas ambiguas, de } \\
\text { aspectos de cohesión textual incorrectos, el manejo } \\
\text { del tono, etc. }\end{array}$ \\
\hline
\end{tabular}

Tabla I. Macrocategorías, categorías y códigos. Fuente: elaboración propia

\section{Resultados y análisis}

En este apartado se presentan los principales hallazgos de cada una de las categorías de análisis encontradas en los discursos verbales escritos de los registros que diligencian los tutores del Centro de Lectura y Escritura (CELEE); a manera de ilustración se exponen ejemplos representativos de estas y se hacen comentarios analíticos a la luz de los referentes teóricos anteriormente desarrollados.

Es importante indicar que se analizaron 154 registros de atención a estudiantes de pregrado y posgrado de las diferentes facultades de la institución: Comunicación Social, Ingeniería, Ciencias Económicas y Administrativas, y Ciencias Básicas. Los estudiantes de posgrado avizoran una posible línea de proyección de trabajo en el Centro, puesto que las necesidades de acompañamiento a los estudiantes de especializaciones y maestrías es alta.

Respecto a la asistencia de los estudiantes al centro, se halló que el porcentaje más alto en el periodo del 2014 correspondió a la Facultad de Comunicación social - I 00 sobre un total de I66-y, en 
el 20 I5, fueron los estudiantes de la Facultad de Ingeniería quienes presentaron una mayor asistencia -72 sobre un total de 162-; al parecer esto se relaciona con las remisiones de los docentes desde los cursos de 'Expresión Oral y Escrita' y 'Seminario de Investigación'. Por su parte, los de Comunicación Social asistieron, principalmente, de los cursos de 'Taller de Comunicación Escrita' y 'Taller de Prensa', 'Comunicación', entre otros. Los estudiantes de la Facultad de Ciencias Económicas y Administrativas venían remitidos, principalmente, de un curso de 'Fundamentos de Contabilidad'.

Los motivos de consulta de los estudiantes son diversos y dependen de la naturaleza del texto que estén construyendo en las asignaturas. En general, consultan más para revisar asuntos relacionados con el proceso de escritura, que con el de lectura. Quienes lo hacen por este último, solicitan orientación sobre estrategias para mejorar la comprensión de textos.

Con respecto a la afluencia al espacio, el número de visitas aumenta significativamente en las semanas de parciales.

\section{A. Macrocategoría: Generalidades (G)}

En primer lugar, de manera general, se identifica que existen diferencias entre los registros de los tutores, debido a que los que son estudiantes tienden a hacer registros más detallados que algunos de los tutores docentes, puesto que señalan las dificultades que tienen los tutorados y las estrategias que utilizan para apoyar los procesos de lectura y escritura. Esto podría estar relacionado con que la comunicación con un igual hace que desaparezcan las fronteras, propiciando un entorno con menos formalismos en el que los alumnos pueden exponer sus dudas a un compañero que ha pasado por una situación similar a la suya ${ }^{12}$. Es significativo mencionar que al tiempo que el tutor-par ayuda a otros, también aprende a tener mayor dominio y profundización en los contenidos trabajados; organiza sus conocimientos para enseñar a otro y tiene mayor conciencia de sus propias carencias, entre otras ${ }^{3}$.

Por otra parte, se observa que son frecuentes los registros centrados en describir la necesidad del estudiante que consulta, pero no se profundiza en el desarrollo de la sesión, ni en los apoyos ofrecidos, los acuerdos definidos u otros aspectos. Ejemplos como "Los estudiantes asistieron para solicitar apoyo en la estructuración de su exposición y de los esquemas a utilizar" o "El estudiante pregunta por la claridad conceptual de la frase, la redacción y si se logra el objetivo de comunicación de la misma." pueden evidenciar que la utilidad del registro es asumida de manera distinta por los tutores, debido a que en estos casos pareciese ser solo un reporte del motivo de consulta de los estudiantes, aun cuando este aspecto se consigna en una columna distinta de la rejilla de registros. Este aspecto hace explícita la necesidad

12 Velasco, P., Domínguez, F., Quintas, S. y Blanco, A. La mentoría entre iguales y el desarrollo de competencias. En IV Jornadas Internacionales Mentoring \& Coaching: Universidad - Empresa. Madrid, España, 2009. Recuperado el 20 de junio de 2015 de http://alfama.sim.ucm.es/wwwisis2/wwwisis.exe/[in=psyke2.in]/?format=breve\&boolean=\%5BNR: 18886604

13 Velasco, P., Blanco, A. y Domínguez, F. Retos de la mentoría en la universidad española. En V Jornadas Internacionales Mentoring \& Coaching: Universidad - Empresa. Madrid, España, 20 I0. Recuperado el 20 de junio de 2015 de http://innovacioneducativa. upm.es/jimcue_10/comunicaciones/2I_Velasco.pdf 
de unificar los criterios para diligenciar este instrumento, ya que hasta el momento no se ha hecho una concertación sobre ello.

Otro de los asuntos que genera inquietud es que algunos registros son exactamente iguales, aun cuando pertenecen a estudiantes distintos, lo que puede mostrar que para algunos de los tutores las atenciones son situaciones generalizables, aunque llama la atención que quienes hacen esto reportan solo lo que el estudiante necesitaba o la actividad que como tutor puso en marcha durante la asesoría; regularmente, en estos registros no se evidencian descripciones más detalladas. Al relacionar esto con las apreciaciones de los párrafos anteriores, se puede identificar una necesidad por definir criterios similares para los registros e, incluso, recordar la ética con la que se debe ejercer el rol de tutor.

\section{B. Macrocategoría:Tendencia de tipo de asesoría (TA)}

En el análisis de los registros se identifican características de tutoría centrada en el tutor y en una relación colaborativa. No se describen rasgos compatibles con la tutoría centrada en el estudiante. A continuación, se presentan ejemplos del primer tipo de asesoría mencionada:

- "Se revisa la redacción del marco teórico de su anteproyecto y se hacen las respectivas correcciones"

- "Se hizo la lectura y se detectaron las dificultades en redacción"

- "Se revisó crónica"

- "Se identificaron algunos pequeños problemas de puntuación pero en general el texto estaba bien escrito."

De los anteriores fragmentos, por la forma en que están escritos, se puede inferir que las tareas de revisión y corrección las asume el tutor, lo que permite cuestionar cuál es el papel que se le otorga al estudiante durante la asesoría y cuál es el aporte que se le está haciendo en términos de estrategias que pueda aplicar, de manera independiente, en situaciones similares a las de consulta. Es evidente que el tutor otorga al estudiante un lugar pasivo en el proceso de aprendizaje, lo que le impide que piense por sí mismo en sus problemas de escritura. Esta situación permitiría visualizar una preocupación más por el texto que por el proceso de aprendizaje del estudiante, tal y como mencionan Alzate-Medina y Peña-Borrero ${ }^{14}$ sobre las características de las asesorías tradicionales.

En la misma línea, Hayward ${ }^{15}$ señala que una de las premisas de un tutor de centro es, en primer lugar, considerar las habilidades de los estudiantes para pensar, crear ideas y consolidar la confianza en su proceso de escritura y, en un lugar secundario, está la revisión de cualquier aspecto del texto (gramática, puntuación y ortografía). En síntesis, los tutores del centro de escritura que dirige Hayward

I4 Alzate, G., y Peña, L. Universitas Phychologica. La tutoría entre iguales: una modalidad para el desarrollo de la escritura en la educación superior, 9, 2010 , págs. 123- 138.

15 Hayward, M. The Writing Center Joumal. Assessing Attitudes Toward the Writing Center, 3, 1983, págs. I-I I. 
afirman que uno de los objetivos básicos para trabajar con los estudiantes es desarrollar su habilidad para pensar y trabajar el lenguaje.

Asimismo, es posible señalar que en esta misma categoría se encuentra que el tutor no asume el texto que trae el estudiante como una posibilidad de trabajar, sino que se espera que con las sugerencias corrija los aspectos señalados.

- "Se sugieren que haga cambios y reordenamiento en varios aspectos."

- "Quedó de escribir los párrafos y traerlos la próxima sesión."

- "Se hizo la lectura y los comentarios, cambios y sugerencias. Se compromete a trabajar en el texto y traerlo para una nueva revisión."

- "Se le dieron explicaciones e ideas para articular los objetivos y la pregunta con el tema de la investigación."

En contraposición, se encuentran algunos reportes escritos que dan cuenta de características más colaborativas de la sesión de asesoría, tal y como se muestra en los siguientes ejemplos:

- "Se explica a la estudiante la importancia de leer en voz alta y se hace una lectura conjunta para identificar la necesidad de modificar tildes, especialmente en verbos en pasado. También se orienta la modificación de algunos aspectos de puntuación, por ejemplo, del manejo de las comas porque la estudiante las usó de forma innecesaria en el texto".

- "El estudiante presenta un fragmento de su texto escrito esperando una retroalimentación sobre la redacción y aspectos estructurales del texto. Así, lee el texto en voz alta y en compañía se discute sobre las intenciones que tenía al redactar cada frase y los signos de puntuación. Finalmente se le ofrece que siga escribiendo durante el tiempo de asesoría para que el avance se discuta al final de ella, y se le invita a regresar de nuevo para seguir el acompañamiento".

Es posible evidenciar que se trata de involucrar explícitamente al estudiante en la dinámica de la asesoría, en estos casos, con la estrategia de lectura en voz alta; de igual manera, se observa que hay una preocupación no solo por aplicar esta estrategia, sino por explicar su uso, lo que puede tener un impacto significativo para el estudiante porque le posibilitaría comprender la utilidad de la misma en la sesión y aplicarla posteriormente para otra tarea. Aparece la metáfora del andamiaje definida por Bruner $^{16}$ con la cual se refiere al carácter necesario de las ayudas que los agentes educativos prestan al aprendiz. Son apoyos transitorios debido a que se retiran de forma progresiva en la medida en que se observa que quien aprende logra control sobre su aprendizaje, en otras palabras, se desechan en la medida en que el tutorado evidencia la resolución de un problema de manera independiente. Se espera, entonces, que el estudiante retome estas estrategias en otras actividades académicas. A diferencia de la tendencia anterior, aquí pareciera que el control de la sesión se intenta compartir entre tutor y tutorado.

16 Bruner, J. Realidad mental y mundos posibles. Barcelona: Ed. Gedisa, 1988. 


\section{Macrocategoría: Estrategias generales de orientación de las asesorías (EOA)}

En los siguientes fragmentos se podrán apreciar ejemplos y comentarios analíticos sobre distintas estrategias usadas de forma frecuente por algunos de los tutores.

\section{Categoría 4: Revisión de la consigna (RC)}

Se solicita a la estudiante explicar "cuál es la consigna del taller para poder orientar la revisión de manera precisa [...]". De este segmento es interesante notar que no solo se pide la consigna, sino que se involucra de manera activa al asesorado para que la revise y la explique de manera oral al tutor. Esta forma de interacción hace parte de compartir el control de la tutoría con el estudiante, como sugiere Brown ${ }^{17}$.

De otra parte, es importante indicar que la revisión de consignas es una estrategia fundamental cuando se entiende la escritura como un proceso que requiere reflexiones y reelaboraciones. A través de esta actividad, los estudiantes pueden reconstruir sus conocimientos y aprender de manera más significativa el contenido y los procesos relacionados con lo que están escribiendo ${ }^{18}$.

\section{Categoría 5: Revisión de material teórico de base (RMB)}

"Se sugiere a la estudiante retomar algunos de los aspectos teóricos que involucra en el taller, puesto que se observa confusión al aplicarlos. Se ejemplifica la identificación de las características del interlocutor y se sugiere revisar todos los demás aspectos del tema en un material que fue entregado por el docente de la asignatura". De este fragmento llama la atención que el tutor tiene la capacidad de identificar que hay confusión al aplicar conceptos teóricos del taller, lo que evidencia que aparte de conocimientos sobre la escritura, estrategias, normas, entre otros, los aspectos conceptuales son igual de necesarios. Al parecer, esta interacción parece indicar que se requiere un trabajo colaborativo con el profesor que tiene el dominio disciplinar, ya que esta situación refiere a saberes en torno a la lectura y escritura como medio para construir los saberes propios de la disciplina.

"Para ampliar sus conocimientos sobre el tema del ensayo se le recomendó la desmitologización de la escritura de Olson y Weston por si quiere ampliar lo de los tipos de argumentos mostrados en outline."; "[... ] le sugerí consultar un texto de Cassany, más la visita al portal del centro de escritura javeriana.". Este segundo fragmento ilustra una de las decisiones más comunes de los tutores, recomendar materiales para que los estudiantes aclaren o amplíen algunos aspectos sobre la tarea que tienen que resolver.

17 Brown, K. Breaking into the tutor"s toolbox: an investigation into strategies used writing center tutorials. Dissertation. University of Louisville, 2008.

I8 Villalobos, J. Lectura y vida. Estrategias de revisión. Estrategias utilizadas por escritores en el nivel universitario, 26, 2005, págs. $42-53$. 


\section{Categoría 6: Conexión entre la tutoría y el espacio de la asignatura (CTA)}

"Los estudiantes acuden para hacer una revisión de la claridad en la redacción de los objetivos del anteproyecto de investigación. Desde el punto de vista de la tutora, la redacción es correcta pero hay dificultades en la formulación del objetivo general porque es ambicioso y dificil de medir. Se establece una conversación para explicar este asunto a los estudiantes y se sugiere consultar con su docente las opciones discutidas en la tutoría". En este fragmento se evidencia una conexión entre la tutoría y el espacio de las asignaturas, lo que es fundamental para que los estudiantes no configuren el Centro como un espacio de "resolución" de todas las problemáticas que puedan presentar sus textos, sino como una oportunidad de diálogo y revisión de estos para salir con otras inquietudes; de esta manera, se aborda la escritura como un proceso y no como un producto cuyas dificultades se resuelven en la inmediatez. Otro de los aspectos que vale la pena destacar de este fragmento es que se delimita claramente cuál es la percepción de la tutora y se intenta negociarla con los estudiantes en lugar de imponerla. De acuerdo con Brown ${ }^{19}$, este es uno de los aspectos que caracteriza las tutorías en las que hay intentos de negociar y equilibrar la participación de tutores y tutorados.

\section{Categoría 7: Explicación breve de algún aspecto (EA)}

"En esta sesión se trabajan aspectos relacionados con la citación de fuentes de acuerdo con normas Icontec porque se observan dificultades con el manejo de estas, especialmente, en las citas directas e indirectas. Se hace una breve explicación de las diferencias entre estas y se apoya la modificación de la redacción de algunos fragmentos". La explicación de estos elementos es común entre los tutores que asesoran estudiantes de trabajo de grado, lo que permitiría identificar una de las posibles líneas de trabajo del CELEE para esta población, proyectando, por ejemplo, ofrecer talleres grupales.

"En primer lugar, se propone al estudiante la definición de palabras claves para iniciar una búsqueda en las bases de datos de la universidad [...]". Es frecuente que los estudiantes acudan al CELEE para recibir asesoría sobre cómo buscar información confiable en la web. En este reporte se aprecia que el tutor intenta guiar la búsqueda de textos desde criterios previamente definidos, lo que podría relacionarse con una concepción de lectura como un proceso estratégico. Hubiese sido interesante que el tutor reportara si explicó la utilidad de esta estrategia para intentar conocer si tiene pretensión de que el estudiante la pueda manejar de forma autónoma en algún momento de su aprendizaje en el Centro. Lo que sí es explícito es que se involucra al estudiante de manera activa al inicio de la sesión.

19 Brown, K. Breaking into the tutor"s toolbox: an investigation into strategies used writing center tutorials. Dissertation. University of Louisville, 2008. 


\section{Categoría 8: Formulación de preguntas a los estudiantes (FP)}

"Los estudiantes acuden a la sesión porque tienen ideas vagas respecto a la redacción del apartado de metodología del artículo de investigación. Se les sugiere retomar la consigna dada por la docente y se identifican los elementos específicos solicitados (método, técnicas y resultados esperados). A través de preguntas orientadoras, se lleva a que los estudiantes formulen las ideas de las etapas de la investigación y se acompaña la redacción de estas". "En la revisión se evidencian algunas contradicciones en el contenido del texto, por lo tanto, se usa la estrategia de formularle preguntas al estudiante para que con base en estas revise y modifique las ideas." En estos fragmentos se destaca la pregunta como un recurso para guiar la discusión y la reflexión para que los tutorados tengan conciencia de las dificultes y sean ellos mismos los que hallen las soluciones. Se observa que el tutor recurre a este recurso para identificar las ideas que tienen los estudiantes sobre el apartado de metodología del artículo de investigación. Se puede apreciar que en este diálogo entre tutor y tutorado sobre el proceso de escritura se hace evidente una tutoría de tipo colaborativa ${ }^{20}$.

\section{Categoría 9: Definición de un plan para la escritura de un texto (DPT)}

"Los estudiantes acuden al espacio en grupo porque están elaborando un anteproyecto y un artículo científico de manera colaborativa. Básicamente, en esta primera asesoría buscan estructurar un plan de trabajo para todo el semestre. La tutora orienta la identificación de los aspectos que los estudiantes requieren trabajar de manera más enfática y se decide en el grupo abordar lo siguiente: citación de acuerdo a normas APA, estructura del artículo (objetivo y estilo de escritura de cada apartado), estrategias de síntesis de información, revisión general de redacción. Los estudiantes se comprometen a acudir a las asesorías cada semana para seguir un proceso alrededor de lo anteriormente mencionado". El segmento anterior es una ilustración que se observa en varios de los reportes, los tutores especifican que trazaron un plan de trabajo en conjunto con los estudiantes, lo que resulta positivo para que las asesorías vayan trascendiendo de manera paulatina la visión remedial, según la cual todas las dificultades de un texto se pueden resolver en el corto tiempo de atención. Es significativo mencionar que en algunos registros se observa que los estudiantes acuden varias veces al Centro. Ante ello surgen inquietudes como ¿Es posible que los estudiantes establezcan relaciones de dependencia? ¿Existe un número de visita límite de un estudiante al Centro? Es bien sabido que la dependencia de los estudiantes no es un aspecto positivo porque no fomenta la responsabilidad frente a su proceso de aprendizaje ${ }^{2 l}$; sin embargo, las tutorías posibilitan un espacio que puede favorecer de forma progresiva este último aspecto, dado que el estudiante puede ir ganando conciencia frente a las dificultades que presenta con los textos pero también de las estrategias que puede poner en juego para superarlas. Así pues, se esperaría que haya, paulatinamente, un control de los procesos de comprensión y producción y comportamientos más autónomos.

20 Alzate, G., y Peña, L. Universitas Phychologica. La tutoría entre iguales: una modalidad para el desarrollo de la escritura en la educación superior, 9, 20 I0, págs. 123- 138.

21 Clark, I. Dialogue in the Lab-Conference: Script Writing and the Training of Writing Lab Tutors, 1982. Recuperado el 10 de mayo de 2015 de http://casebuilder.rhet.ualr.edu/wcrp/publications/wcj/wcj2.I/wcj2. I_clark.pdf 


\section{Categoría 10: Ofreciendo modelos de textos (OMT)}

"Durante la sesión se hizo la búsqueda de un texto para modelar algunas estrategias de lectura. Se sugirió al estudiante trabajar lectura del resumen del texto para evaluar si era pertinente o no para su tarea. Una vez él mismo reconoció la utilidad, se propuso lectura detallada de los subtítulos que consideraba más importantes y parafraseo de la información para incluir en el marco conceptual". Este fragmento da cuenta de que se identifica necesidad de involucrar de manera activa al estudiante en la sesión y entender que el proceso de lectura puede tener pasos o estrategias que se "modelan" al estudiante. Esto tiene relación con el modelo socio-cognitivo de aproximación a la lectura y la escritura, en el que se reconoce la importancia de estrategias de modelamiento. De acuerdo con Zimmerman y Kitsantas ${ }^{22}$, uno de los elementos fundamentales en el proceso de aprendizaje es la presencia de los otros para la consecución de la autorregulación, ya afirman que las actividades modeladas y de realimentación son guías fundamentales para los aprendices, quienes deben avanzar hacia la interiorización y automanejo de los procesos modelados para que sean capaces de aplicarlos en diferentes situaciones. Para estos autores los niveles de observación y emulación constituyen experiencias de aprendizaje social que favorecen las competencias de autocontrol y autorregulación.

\section{Categoría I I: Lectura en voz alta (LVA)}

"A medida que el estudiante avanza en la lectura en voz alta, identifica dificultades de redundancia de información y falta de cohesión en general. [... ] logra evidenciar estas falencias y se discuten posibilidades de redacción que concreten más las ideas." En este fragmento se observa que la lectura en voz alta posibilita al estudiante reflexionar, confirmar hipótesis, verbalizar sus inquietudes y tomar conciencia de sus conocimientos y de las dificultades en la escritura del texto para solucionarlas. Esta estrategia permite que los tutorados se responsabilicen de su propio proceso de aprendizaje y traten de asumir el control como escritores.

Esta es una de las estrategias de revisión de textos más usada en dinámicas que requieran interacción, tal y como sucede en las asesorías presenciales de los centros de escritura. Su importancia radica en que permite acceder al contenido del texto de manera simultánea para todos los participantes. Leer en voz alta puede facilitar una realimentación auditiva de la forma como se ha textualizado el contenido del escrito, que puede, a su vez, favorecer la identificación de ideas ambiguas, de detectar aspectos de cohesión, coherencia, manejo del tono, entre otros.

Todas las estrategias descritas anteriormente muestran que, en general, la mayoría de los tutores reportan de manera explícita las estrategias a través de las que intentan involucrar de manera activa al estudiante en la sesión. Esto es interesante porque se trasciende la visión remedial del texto para

22 Zimmerman B. J. y Kitsantas, A. Journal of Educational Psychology. Acquiring writing revision and self-regulatory skill through observation and emulation, 94, 2002, págs. 660-668. 
aportar elementos que posibiliten un abordaje estratégico de los procesos de lectura y de escritura por parte de los estudiantes.

\section{Conclusiones}

Esta caracterización inicial permite esbozar la necesidad de socializar y compartir, entre los integrantes del equipo de trabajo del Centro de Lectura y Escritura de la institución, las visiones que posibilitan su funcionamiento. Las concepciones de lectura y escritura, de la tutoría como forma de acompañamiento, de las estrategias de orientación, entre otros elementos, se encuentran especificados en los documentos internos del Centro; no obstante, no son conocidos por el equipo de tutores, asunto de suma importancia, ya que estos recursos representan la filosofía que se necesita proyectar para posicionar el Centro a nivel institucional.

De acuerdo con los reportes escritos, se podría afirmar que la mayoría de tutores del CELEE pone en marcha distintas acciones para orientar la lectura y la escritura como procesos, más que como actividades automáticas, e intentan ejemplificar en las asesorías formas de acceder a los textos de manera estratégica. De igual manera, se puede evidenciar que predomina una tendencia de tutoría colaborativa, aunque también se identifican rasgos de asesoría centrada en el tutor. Esto vislumbra una necesidad por propiciar espacios de encuentro de saberes entre los tutores para que sean ellos mismos quienes comenten qué tipo de estrategias usan y qué resultados obtienen; de esta forma, podría configurarse de manera precisa el perfil no solo del tutor que hace parte del CELEE, sino también dimensionar la importancia de la precisión en los registros escritos de atención porque, hasta el momento, son el único insumo para conocer cómo funciona el espacio.

Ahora bien, las categorías organizadas para este ejercicio investigativo permitieron hacer una aproximación al estudio de las dinámicas que se ponen en juego en las tutorías en lectura y en escritura, y hacer aportes en términos de evidenciar posibles líneas de análisis. En este sentido, esta exploración representa un aporte interesante para continuar profundizando sobre la importancia de la interacción y los encuentros con otros para efectos de mejorar los procesos de lectura y de escritura, y sobre los elementos pedagógicos que median lo que ocurre en un lugar en el que se exploran alternativas para aportar al desempeño académico de los estudiantes.

\section{Bibliografía}

Alzate, G., y Peña, L. Universitas Phychologica. La tutoría entre iguales: una modalidad para el desarrollo de la escritura en la educación superior, 9, 2010, págs. 123- 138.

Brown, K. Breaking into the tutor"s toolbox: an investigation into strategies used writing center tutorials. Dissertation. University of Louisville, 2008.

Bruner, J. Realidad mental y mundos posibles. Barcelona: Ed. Gedisa, 1988. 
Clark, I. Dialogue in the Lab-Conference: Script Writing and the Training of Writing Lab Tutors, 1982. Recuperado el 10 de mayo de 2015 de http://casebuilder.rhet.ualr.edu/wcrp/publications/wcj/wcj2.l/wcj2.I clark.pdf

Hayward, M. The Writing Center Joumal. Assessing Attitudes Toward the Writing Center. 3, 1983, págs. I-I I.

Rodríguez, G., Gil Flores, J. y García Jiménez, E. Metodología de la investigación Cualitativa. Málaga: Aljibe, 1999.

Velasco, P., Blanco, A. y Domínguez, F. Retos de la mentoría en la universidad española. En V Jornadas Internacionales Mentoring \& Coaching: Universidad - Empresa. Madrid, España, 20 I0. Recuperado el 20 de junio de 2015 de http://innovacioneducativa.upm.es/jimcue_I0/comunicaciones/2I_Velasco.pdf

Velasco, P., Domínguez, F., Quintas, S. y Blanco, A. La mentoría entre iguales y el desarrollo de competencias. En IV Jornadas Internacionales Mentoring \& Coaching: Universidad - Empresa. Madrid, España, 2009. Recuperado el 20 de junio de 2015 de http://alfama.sim.ucm.es/wwwisis2/wwwisis.exe/[in=psyke2.in]/?format=breve\&b oolean=\%5BNR: 8886604

Villalobos, J. Lectura y vida. Estrategias de revisión. Estrategias utilizadas por escritores en el nivel universitario, 26, 2005, págs. 42-53.

Zimmerman B. J. y Kitsantas, A. Journal of Educational Psychology. Acquiring writing revision and self-regulatory skill through observation and emulation, 94, 2002, págs. 660-668. 